\title{
Galaxie Tennis: Un estudio de caso
}

\author{
Bernard Pestre
}

Federación Francesa de Tenis

RESUMEN

La enseñanza del tenis, como toda otra enseñanza, ha evolucionado considerablemente desde los años 70 cuando no era raro ver hasta veinte jugadores, niños o adultos, en la misma cancha, delante de un instructor, imitando los golpes de tenis, sin ni siquiera golpear una pelota o pelotear con un compañero. Gradualmente, con la idea de que la técnica no es más que un medio para un fin, el juego de tenis ha ganado terreno. Hoy, en la mayoría de las escuelas de tenis del mundo, podemos ver a los niños "jugando tenis" desde las primeras sesiones. Esto va de la mano con la evolución de la sociedad y con el estilo de enseñanza orientada a lo individual - "democrático", empático e interactivo (Reid, Crespo, 2009), que prevalece ahora en la enseñanza deportiva.
Palabras clave: Desarrollo, juego y partidos, adquisición de habilidades

Recibido: 15 May 2017

Aceptado: 20 Jul 2017

Autor correspondiente:

Bernard Pestre, Federación

Francesa de Tenis.

Correo electrónico:

bpestre@fft.fr

\section{INTRODUCCIÓN}

\section{Orígenes del programa "Galaxie"}

En 2014, la Federación Francesa de Tenis (FFT) inició un programa totalmente nuevo para las escuelas de tenis, llamado "Galaxie Tennis". El objetivo del programa es mantener a los niños en los clubes, fomentando para que jueguen tenis competitivo, con un logo que es una evocación del juego, la competición y la velocidad de éste.

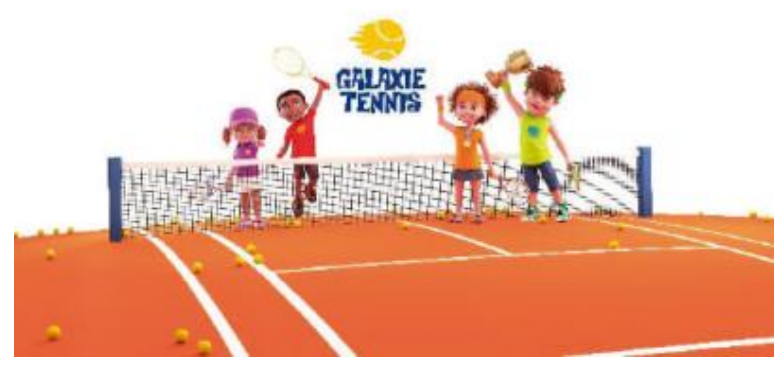

Figura 1. Lou, Zoé, Arthur y Hugo son los cuatro personajes que representan los diferentes grupos de edades.

El concepto es muy similar al del programa "Play and Stay" de la ITF. Para mantenerse en línea con la ITF, la FFT decidió conservar las etapas Roja, Naranja, Verde y Amarilla, que indican el progreso del niño. Todos estos elementos están incluidos en el manual del profesor (2014). Se agregaron dos etapas para asegurar la adquisición de los elementos técnicos y tácticos iniciales.

- En la cancha de etapa Blanca de $8 \mathrm{~m}$., la pelota rueda en el suelo, sin red.

- En la cancha de etapa Púrpura de11 m., se juega con las pelotas "Le Petit Tennis", utilizando el ancho de la cancha, y con una red de $0,5 \mathrm{~m}$.

Los formatos siguientes son idénticos a los de "Play and Stay".

- El Juego en etapa Roja se juega en los cuadros de saque, utilizando pelotas de fieltro o de espuma de goma, bajando la red lo más posible, (altura máxima 0,8 m).

- El Juego en etapa Naranja se juega en cancha de $18 \mathrm{~m}$. con pelota naranja; la altura de la red será de $0.8 \mathrm{~m}$. Cabe mencionar que la FFT recomienda el uso del ancho completo de la cancha de individuales.

- El Juego en etapa Verde se juega en cancha de 23,77 m. con pelota verde; la altura de la red será estándar.

Cada etapa de aprendizaje se caracteriza por las adquisiciones tácticas, técnicas y conductuales.

Estas adquisiciones son validadas por el profesor durante los llamados días de "Juego y Partidos", pues, tanto el juego como el contrincante son los dos factores que servirán de guía para las contribuciones técnicas que harán los profesores a los niños. Organizados como parte del programa de tenis para los 
colegios, estos eventos de un día de "Juego y Partidos" son fundamentales por muchas razones.

\section{DESAFÍOS}

No es ningún secreto que la FFT, como muchas otras asociaciones nacionales de tenis, está sufriendo la disminución del número de jóvenes participantes, desde hace ya algunos años. Esta situación se puede explicar por los siguientes factores:

- La baja tasa de retención de jóvenes en los programa de tenis para los colegios: sólo el $63 \%$ de los miembros de entre 5 y 10 años de edad en 2015 renovaron su membrecía en 2016. Si bien algunas regiones tienen una tasa de retención superior al $75 \%$, otras no llegan al $45 \%$. Las estadísticas relacionadas con las niñas son aún más alarmantes que las de los niños, pues su retención es un $10 \%$ menor.

- Esta disminución en la renovación de la membrecía ya no se compensa reclutando nuevos jóvenes. El programa "Galaxie Tennis" no intenta solucionar todos los problemas. Sin embargo, estamos convencidos de que la retención de los jóvenes está inextricablemente ligada al progreso logrado y, por lo tanto, depende de la calidad de la enseñanza. La filosofía de enseñanza de "Galaxie Tennis" se basa en el uso adecuado de las canchas Blancas, Púrpura, Rojas, Naranja y Verdes. Por "adecuado" entendemos que el material (pelotas y raquetas) debe adaptarse a cada tipo de cancha. Además, es fundamental atenerse a las habilidades que deben necesariamente adquirirse.

\section{GENERALIDADES DE LOS DÍAS DE "JUEGO Y PARTIDOS"}

- Estos eventos crean un entorno favorable para la introducción de los niños a la competición. Dependiendo de su nivel, aprenden a reconocer la diferencia entre una pelota "dentro" y una pelota "fuera", y luego, a contar los puntos, jugar una muerte súbita, etc. Pero el propósito principal de estos eventos de un día es dar a los niños la oportunidad de jugar varios partidos cortos y de competir con otros.

- Estos días sirven también para destacar las habilidades adquiridas durante el ciclo de enseñanza que acaba de terminar, y empoderar a los jóvenes jugadores, animándoles para que continúen mejorando. De este modo se puede completar el ciclo (Blanco, Púrpura, Rojo, Naranja o Verde).

- También permite que los niños tengan una clasificación de acuerdo con los estándares nacionales; de este modo, pueden participar en talleres organizados para niños y niñas de nivel similar o en competiciones oficiales, comenzando desde la etapa Naranja.
- Todos los niños reciben, de parte de su profesor, un pasaporte que mantendrán a lo largo de su experiencia "Galaxie", desde la cancha Blanca hasta la Verde. El profesor completa el pasaporte seleccionando las diferentes habilidades adquiridas al concluir cada día de "Juego y Partidos". Cada vez que un niño progresa a la etapa siguiente, la persona a cargo en el club, lo registra en el sistema de gestión nacional de clubes de tenis.

La enseñanza cuidadosa, el juego de partidos, la evaluación de las habilidades y el sistema de pasaportes son los componentes clave del programa. Si el club se ajusta estrictamente a los principios "Galaxie", los niños y sus padres se integrarán. Varios estudios demuestran que la competición adaptada, cuando se presenta correctamente, es la mejor manera de motivar a los niños para continuar con una actividad. Una introducción temprana y divertida al juego de partidos logra que la transición hacia la competición sea más suave y más fácil para los niños. Esto es particularmente verdadero en el caso de las niñas, a quienes no les gusta compararse con sus amigas (Renoult, 2014).

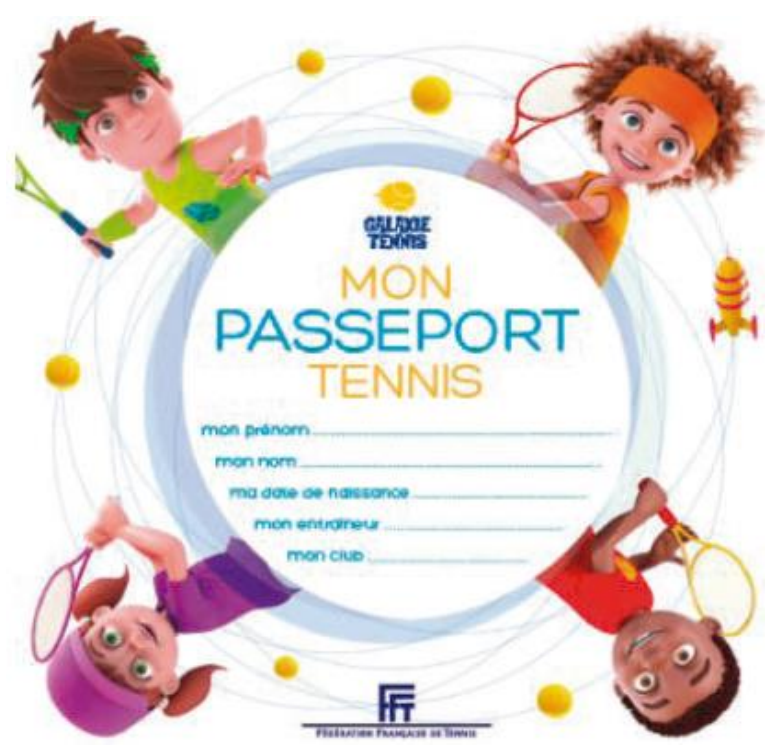

Figura 2. Ejemplo del pasaporte que se distribuye en las escuelas de tenis.

Los niños avanzan a la etapa siguiente cuando se han adquirido las habilidades establecidas en la etapa en la que se encuentran.

Seguidamente presentamos algunos ejemplos de situaciones de partido sugeridas, que ayudan a los entrenadores a validar, si se han logrado las habilidades necesarias.

\section{Etapa blanca}

Cancha de $8 \mathrm{~m}$ / Pelota blanca inflable rodando en el piso / El medio de la cancha es visible en el suelo.

Primero a 3 goles / Duración máxima: 5 min. 


\section{Etapa púrpura}

Cancha de 11 m. / Pelota púrpura "Le Petit Tennis" / Primer servicio sobre la cabeza / Servicio alternado.

Primero a 7 puntos / Duración máxima del partido: 9 min.

\section{Etapa roja}

Cancha de 12,8 m (cuadros de saque) / Pelotas de fieltro o de espuma de goma.

Juego de individuales - 2 canchas contiguas: 4 jugadores en media cancha/ 2 jueces/ 1 muerte súbita/ Duración máxima del partido: $10 \mathrm{~min}$.

Juego de dobles - 1 cancha / 4 jugadores / 1 juez / 2 muertes súbitas / Al menos 3 rotaciones / Mínimo 2 partidos por equipo.

\section{Etapa naranja}

Cancha de 18 m / Pelota Naranja.

Juego de individuales - Primero a 2 juegos: punto decisivo en 40 iguales; muerte súbita en 1-1.

Máxima duración de los partidos: 15 min.

Juego de dobles - 1 cancha (sin líneas paralelas).

Primero a 2 juegos: punto decisivo en 40 iguales; muerte súbita en 1-1.

3 rotaciones de 15 min. cada una.

\section{Etapa verde}

Cancha de 23,77 m / Pelota Verde.

Juego de individuales- Los jugadores son responsables de cantar los puntos / partidos de 1 set: primero a 4 juegos/ punto decisivo en 40 iguales/ muerte súbita en 3-3.

Juego de Dobles- 1 cancha sin líneas paralelas/ Los jugadores son responsables de cantar los puntos / 2 sets de 4 juegos/ punto decisivo en 40 iguales/ muerte súbita en 3-3.

En cada etapa, el entrenador verifica si el niño ha desarrollado las habilidades necesarias en las 4 áreas siguientes:

- Conducta

- Reglas y puntaje

- Elementos tácticos

- Elementos técnicos

A continuación se describen las habilidades que necesitan desarrollar los niños para avanzar a la etapa siguiente.

VER ANEXO I

\section{CONCLUSIÓN}

El desarrollo del tenis depende de la habilidad de mantener a los niños después de que se asocian a un club. La atmósfera creada por los dirigentes del club y la calidad de las instalaciones contribuirán, obviamente, a la diversión de los niños que pasarán largas horas con amigos, pero la clave para desarrollar un interés duradero por el tenis será siempre la sensación de mejorar y de transformarse en un "real" tenista. La temprana introducción al juego de partidos resta importancia a la competición. Jugar muchos partidos con amigos del mismo grupo, y luego, de la misma escuela de tenis, proporciona a los jugadores jóvenes la oportunidad de ver su progreso y de competir con otros sin sentir demasiado estrés.

\section{REFERENCIAS}

Crespo, M, Reid, M. (2009) : Enseigner le tennis aux joueurs débutants et intermédiaires.

Delaigue, N, Blanco, J-F, de Castilla, H, Kronenberger, A, Renoult, M, Pestre, B. (2014) : Le cahier de l'enseignant.

Renoult, M. (2014) : I'emag du Club fédéral des enseignants. http:// www.siege.fft.fr/cfe/cfe_d/data_1/pdf/aa/aaemag96.pdf

\section{CONTENIDO ITF ACADEMY RECOMENDADO (HAZ CLICK ABAJO)}

\section{ITF Academy}

Derechos de Autor (c) 2017 Bernard Pestre.

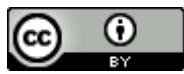

Este texto está protegido por una licencia CreativeCommons 4.0

Usted es libre para Compartir -copiar y redistribuir el material en cualquier medio o formato- y Adaptar el documento -remezclar, transformar y crear a partir del material- para cualquier propósito, , incluso para fines comerciales, siempre que cumpla la condición de:

Atribución: Usted debe dar crédito a la obra original de manera adecuada, proporcionar un enlace a la licencia, e indicar si se han realizado cambios. Puede hacerlo en cualquier forma razonable, pero no de forma tal que sugiera que tiene el apoyo del licenciante o lo recibe por el uso que hace de la obra. 


\begin{tabular}{|c|c|c|c|c|c|c|}
\hline & & BLANCA & PÜRPURA & houx & NLARANUA & VEROE \\
\hline \multicolumn{7}{|c|}{$\begin{array}{l}\text { TRANSICION DE UNA ETAPA A LA SIGUIENTE: EL NIFO DENUESTRA QUE PUEOE.. } \\
\text { Nota: Toda habilidad adquirida en una cancha debe cDntinuar en las canchas siguientes. }\end{array}$} \\
\hline \multicolumn{2}{|c|}{ CONDUCTA } & $\begin{array}{l}\text { Tatrecher la mano } \\
\text { ded actuerzario (y } \\
\text { def juex) al finalizar } \\
\text { el partibo. }\end{array}$ & $\begin{array}{l}\text { Cantar en vor aib las } \\
\text { pelotas "fuera" y el } \\
\text { puntaje }\end{array}$ & & $\begin{array}{l}\text { Arreglar las } \\
\text { disputas } \\
\text { potenciales con su } \\
\text { adversario }\end{array}$ & $\begin{array}{l}\text { Respetar at } \\
\text { adversario v } \\
\text { muntener ina } \\
\text { conducta apropisda } \\
\text { en todo momenta }\end{array}$ \\
\hline \multirow{2}{*}{\multicolumn{2}{|c|}{ MEGLAS Y PUNTAE }} & $\begin{array}{l}\text { Esperar hesta que } \\
\text { el otro jugador esté } \\
\text { listo antes de } \\
\text { comenuar el punto }\end{array}$ & $\begin{array}{l}\text { Identificar la } \\
\text { diferencia entre una } \\
\text { pelota "dentra" y } \\
\text { una Toera" }\end{array}$ & \multirow[t]{2}{*}{$\begin{array}{l}\text { fugar una muerte } \\
\text { subitita }\end{array}$} & \multirow[t]{2}{*}{$\begin{array}{l}\text { Contar ios puntos } \\
\text { en modo "juego" }\end{array}$} & \multirow[t]{2}{*}{$\begin{array}{l}\text { Aplicar las reglas } \\
\text { independientemente }\end{array}$} \\
\hline & & $\begin{array}{l}\text { Turnasse para } \\
\text { iniriair bos puntas }\end{array}$ & $\begin{array}{l}\text { Uever y recondar el } \\
\text { puntaje }\end{array}$ & & & \\
\hline \multirow{7}{*}{\multicolumn{2}{|c|}{$\begin{array}{l}\text { ELENeENTOS } \\
\text { TACTicos }\end{array}$}} & $\begin{array}{l}\text { Tomar la posición al } \\
\text { iniciar el pusto }\end{array}$ & $\begin{array}{l}\text { Golpear varios } \\
\text { puntos } \\
\text { sucrelvamente }\end{array}$ & $\begin{array}{l}\text { Moves al adversariso } \\
\text { por todfa le cancha }\end{array}$ & $\begin{array}{l}\text { Atacar jugando cen } \\
\text { mavar velocidad }\end{array}$ & Utilizar ef mejor tiro \\
\hline & & $\begin{array}{l}\text { Enviar la pelota all } \\
\text { lugar donde no esté } \\
\text { ef adversario }\end{array}$ & & Ejecutar voleas & $\begin{array}{l}\text { Defendes hariendo } \\
\text { el juego más lento }\end{array}$ & \\
\hline & & $\begin{array}{l}\text { Recuperar la } \\
\text { posicidin en la } \\
\text { cancha }\end{array}$ & & & $\begin{array}{l}\text { Utilizar diferentes } \\
\text { efectos }\end{array}$ & \\
\hline & & & & & $\begin{array}{l}\text { Efectuar el ler } \\
\text { saque con mayar } \\
\text { velocidiod }\end{array}$ & \\
\hline & & & & & $\begin{array}{l}\text { Ser agresiva en el } \\
20 \text { tiro }\end{array}$ & \\
\hline & & & & & $\begin{array}{l}\text { Tomar la posición af } \\
\text { iniclar el punto en } \\
\text { Indhiduales }\end{array}$ & \\
\hline & & & & & $\begin{array}{l}\text { Tomar la posición al } \\
\text { iniciar el punto en } \\
\text { dobles }\end{array}$ & $\begin{array}{l}\text { Critarsey "robar" } \\
\text { en dohles }\end{array}$ \\
\hline \multirow{2}{*}{\multicolumn{2}{|c|}{ Servicio }} & & $\begin{array}{l}\text { Adoptar la posición } \\
\text { cesrada }\end{array}$ & $\begin{array}{l}\text { Senerir utilizandia ta } \\
\text { posición de trofieo }\end{array}$ & Servir cona efecto & $\begin{array}{l}\text { Ejecuitar un } 20 \\
\text { servicio eartado o } \\
\text { liftado }\end{array}$ \\
\hline & & & $\begin{array}{l}\text { Golpear la peiota } \\
\text { con remote }\end{array}$ & $\begin{array}{l}\text { Martener el } \\
\text { envilibrio } \\
\end{array}$ & & \\
\hline \multirow{6}{*}{$\begin{array}{l}\text { ELEMENTOS } \\
\text { TÉCNacos }\end{array}$} & \multirow{4}{*}{$\begin{array}{l}\text { luevos } \\
\text { de } \\
\text { fonda }\end{array}$} & $\begin{array}{l}\text { Sostener la raqueta } \\
\text { en el extremo ded } \\
\text { mange. }\end{array}$ & $\begin{array}{l}\text { Adoptar una } \\
\text { ponición de esperia } \\
\text { para la devoludidin } \\
\text { det servicio con la } \\
\text { mano libre en la } \\
\text { garganta de la } \\
\text { ragieta }\end{array}$ & $\begin{array}{l}\text { Sostenes la raquets } \\
\text { con amban mancs } \\
\text { entre las tiros }\end{array}$ & 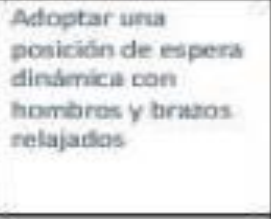 & \\
\hline & & $\begin{array}{l}\text { Sostenes la raqueta } \\
\text { con ambas manos } \\
\text { al comentrar el } \\
\text { gunto }\end{array}$ & & $\begin{array}{l}\text { Diferenciser las } \\
\text { empuañadiura de } \\
\text { derrecha y de revels }\end{array}$ & & \multirow[b]{2}{*}{$\begin{array}{l}\text { Efectuar la } \\
\text { serminación def tiro }\end{array}$} \\
\hline & & $\begin{array}{l}\text { Juftar los galpes de } \\
\text { derecha y de revits } \\
\text { utilizanda ambos } \\
\text { lados de la cara de } \\
\text { ta raqueta }\end{array}$ & $\begin{array}{l}\text { Golpear la pelota } \\
\text { delante del cuerpo } \\
\text { en el golpe de } \\
\text { deredha }\end{array}$ & $\begin{array}{l}\text { Preparar el golpie } \\
\text { de dernecha con la } \\
\text { cabeza de fas } \\
\text { raqueta sotbre la } \\
\text { mano }\end{array}$ & & \\
\hline & & $\begin{array}{l}\text { Golpear tanto reviss } \\
\text { a uns mano como } \\
\text { revés a dos manos }\end{array}$ & $\begin{array}{l}\text { Golpear la peloca } \\
\text { delante del cuerpo } \\
\text { en el nevies }\end{array}$ & $\begin{array}{l}\text { Preparar ef golpe } \\
\text { de derecha con la } \\
\text { cabera de la. } \\
\text { raqueta sotbre la } \\
\text { mano } \\
\end{array}$ & & \\
\hline & $\begin{array}{l}\text { Jueso } \\
\text { de red }\end{array}$ & & & $\begin{array}{l}\text { Goloear la pelota } \\
\text { detante. }\end{array}$ & $\begin{array}{l}\text { Golpear ta peiotas } \\
\text { delante avanuando }\end{array}$ & \\
\hline & $\begin{array}{l}\text { Juego } \\
\text { de pies }\end{array}$ & $\begin{array}{l}\text { Acomodarse para ia } \\
\text { pelota entrante }\end{array}$ & $\begin{array}{l}\text { Golpear } \\
\text { manteniendo la } \\
\text { estabilidad }\end{array}$ & $\begin{array}{l}\text { Mantenerse } \\
\text { dinainico entre } \\
\text { tinas }\end{array}$ & $\begin{array}{l}\text { Deslixar en tierra } \\
\text { batida }\end{array}$ & $\begin{array}{l}\text { Acelerar / } \\
\text { desucelerar }\end{array}$ \\
\hline
\end{tabular}

